\title{
Collisions of solitons and vortex rings in cylindrical Bose-Einstein condensates
}

\author{
Stavros Komineas ${ }^{1}$ and Joachim Brand ${ }^{2}$ \\ 1 TCM Group, Cavendish Laboratory, Madingley Road, Cambridge CB3 OHE, United Kingdom. \\ ${ }^{2}$ Max Planck Institute for the Physics of Complex Systems, Nöthnitzer Straße 38, 01187 Dresden, Germany
}

(Dated: October 29, 2018)

\begin{abstract}
Interactions of solitary waves in a cylindrically confined Bose-Einstein condensate are investigated by simulating their head-on collisions. Slow vortex rings and fast solitons are found to collide elastically contrary to the situation in the three-dimensional homogeneous Bose gas. Strongly inelastic collisions are absent for low density condensates but occur at higher densities for intermediate velocities. The scattering behaviour is rationalised by use of dispersion diagrams. During inelastic collisions, spherical shell-like structures of low density are formed and they eventually decay into depletion droplets with solitary wave features. The relation to similar shells observed in a recent experiment [Ginsberg et al. Phys Rev. Lett. 94, 040403 (2005)] is discussed.

PACS numbers: 03.75.Lm, 47.32.Cc, 47.37.+q
\end{abstract}

The observation of dark solitons and vortex rings in a series of experiments in Bose-Einstein condensates (BECs) 1, 2, 3, 4] has shown that this is an excellent physical system for the study of nonlinear waves. Theoretical work has shown that the latter have the form of solitons, vortex rings, and solitonic vortices [ $[, 6,6,7,8]$. In the recent experiment of Ginsberg et al. [9] collisions between solitary waves were observed. While vortex rings and solitons were robust in many collision events, in some cases shell structures of low particle density were observed, which had not been predicted before.

In the one-dimensional (1D) nonlinear Schrödinger equation (NLS), which describes the 1D Bose gas, soliton collisions are elastic, that is no energy is radiated and the outgoing solitons are the same as the colliding ones [10]. In the homogeneous three-dimensional (3D) Bose gas, solitary waves have the form of vortex rings or rarefaction pulses 11. Their interactions are important for the understanding of superfluid turbulence. In contrast to the 1D case, collisions are generally inelastic. Large vortex rings annihilate when they collide head-on by increasing their radius and radiating phonons 12,13 ]. Collisions at oblique angles or with an impact parameter result in vortex-line reconnections or produce Kelvin-wave radiation [14, 15]. Solitary wave collisions in trapped 2D systems were previously considered in Refs. [16, 17].

A model system that connects the 1D and 3D solitary waves is given by the cylindrically confined BEC. Studying elementary collision processes in this model is thus of fundamental theoretical interest and furthermore it leads to a deeper understanding of the experimentally observed structures.

In this Letter we present a detailed numerical and theoretical study of head-on collisions between solitary waves in a cylindrically confined BEC. We test the robustness of solitary waves when they interact. The detailed behaviour of solitary waves under collision for low density condensates is found to be similar to soliton dynamics in the NLS [10]. This is, however, only a limiting case

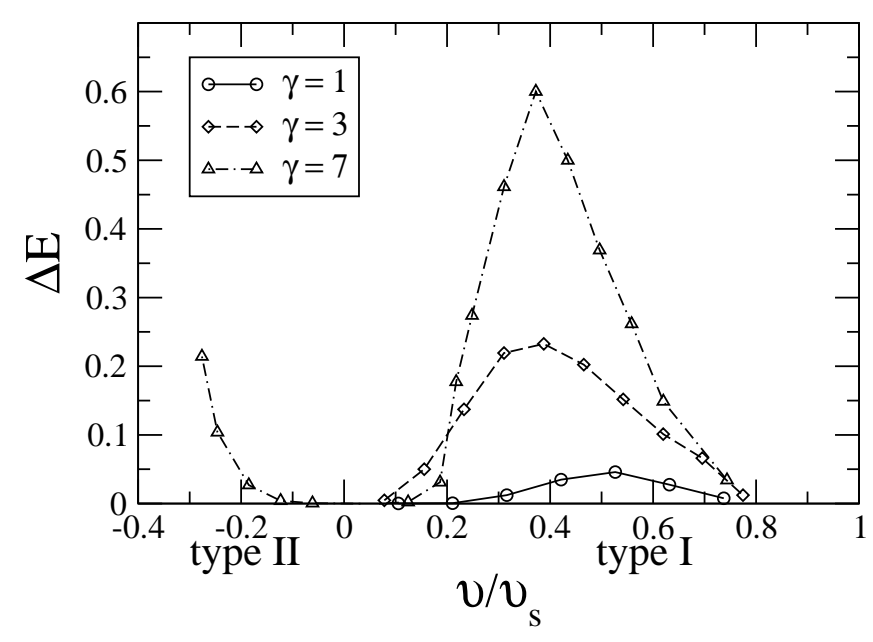

FIG. 1: The energy fraction $\Delta E$, defined in (4), which is radiated during collision of solitons and vortex rings of type I and II (opposite direction) as a function of their initial velocity, for three values of the coupling constant $\gamma=n_{1} a$. The velocity is normalised to the speed of sound $v_{s}=0.95,1.29,1.61$ (in units of $\left.\hbar /\left(m l_{\rho}\right)\right)$ for $\gamma=1,3,7$, respectively. The symbols correspond to the simulations performed.

for the present system, which also shows more complicated dynamical behaviour. For higher particle densities we find that the collision dynamics of solitary waves, including vortex rings, is very different than in the homogeneous 3D Bose gas. As shown in Fig. 11 we find elastic collisions for small and large velocities. At intermediate energies, however, inelastic collisions often produce temporary spherical shells reminiscent of those observed experimentally [9]. In the following, we introduce the model and discuss the various regimes in detail.

We assume a cylindrical and infinitely-elongated trap as in Ref. [] with symmetry axis z. The Gross-Pitaevskii equation can be written in the dimensionless form

$$
i \frac{\partial \Psi}{\partial t}=-\frac{1}{2} \Delta \Psi+\frac{1}{2} \rho^{2} \Psi+4 \pi \gamma|\Psi|^{2} \Psi,
$$



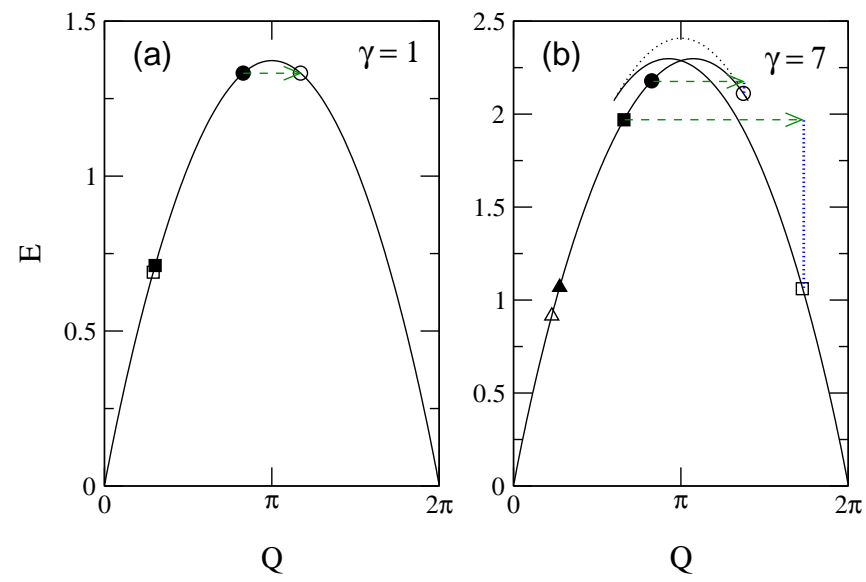

FIG. 2: The excitation energy $E$ in units of $n_{1} \hbar^{2} /\left(m l_{\rho}\right)$ versus impulse $Q$ in units of $n_{1} \hbar$ for axially symmetric solitary waves [7]. At $\gamma=1$ in panel (a) there is one family of solitons. Panel (b) for $\gamma=7$ shows two fundamental branches (solid lines) of vortex rings related by (3). The vortex ring radius has a minimum at the cusps, where $|v|=0.3 v_{s}$. The impulse transfer during bounce-off collisions is indicated by the dashed arrow connecting initial solitary waves (filled symbols, see text) with collision products (open symbols). The vertical dotted lines indicate the energy loss.

where $\rho=\sqrt{x^{2}+y^{2}}$ is the radial coordinate. The dimensionless coupling constant $\gamma \equiv n_{1} a$ is the only parameter entering the equation, where $a$ is the scattering length and $n_{1}$ is the linear particle density along the symmetry axis z. Length is measured in units of the oscillator length $l_{\rho}=\sqrt{\hbar / m \omega_{\rho}}$, where $m$ is the atomic mass and $\omega_{\rho}$ is the transverse trapping frequency. The unit of time is $1 / \omega_{\rho}$. At $z \rightarrow \pm \infty$ the wave function approaches the ground state in the transverse plane with $\partial \Psi / \partial z=0$ and $\int|\Psi|^{2} d^{2} x=1$.

The wave functions of solitary waves in the cylindrical trap are of the form

$$
\Psi(x, y, z, t ; v)=\psi(x, y, z-v t) e^{-i \mu t},
$$

where $v$ is the velocity of propagation and $\mu$ the chemical potential. For $\gamma \lesssim 1.5$ only one family of solitary waves exists with velocities $-v_{s}<v<v_{s}$. They are related to the dark soliton of the NLS. We show the energymomentum dispersion for $\gamma=1 \mathrm{in} \mathrm{Fig.} \mathrm{2a} \mathrm{7].} \mathrm{A} \mathrm{modified}$ momentum $Q$, called the impulse, has been defined so that the velocity is given by the slope of the curve $v=$ $d E / d Q$ [7]. Solitons with opposite velocities are obtained by the symmetry transformation

$$
\Psi \rightarrow \Psi^{*}, v \rightarrow-v,
$$

where the star denotes complex conjugation.

We numerically simulate the elementary process of a head-on collision of two solitons at $\gamma=1$ with wave functions related by Eq. (3). We assume axial symmetry throughout the collision process and we typically use a

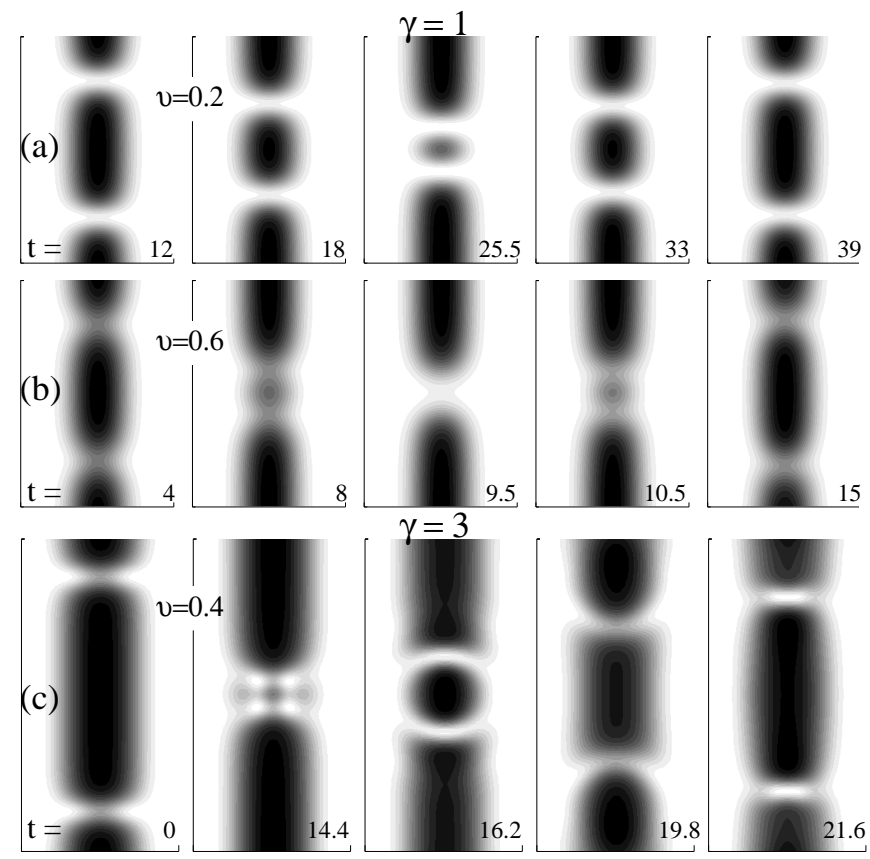

FIG. 3: Plots of the particle density $|\Psi|^{2}$ for solitary wave collisions on a scale from white at zero to black at maximum density for each plot. Each row shows five snapshots of a solitary wave collision with values of $\gamma=1(\mathrm{a}, \mathrm{b})$, and $\gamma=3$ (c), and velocities $v$ as indicated. The time for each snapshot is shown in the figure. The spatial dimensions of the plots are $8 \times 12$ for $(\mathrm{a}, \mathrm{b})$ and $8 \times 16$ for $(\mathrm{c})$. The initial separation (at time $t=0$ ) of the solitary waves is $d=12$ in units of $l_{\rho}$ for all cases.

lattice size $6 \times 60$ in the $(\rho, z)$ plane and a lattice spacing 0.1 for both variables. We integrate Eq. (1) in time using a Runge-Kutta method for various values of velocity $v$ of the initial solitons each calculated as in Ref. [8]. The outgoing solitons after collision are in all cases very similar to the incoming ones and no visible radiation is produced, thus collisions are elastic. A measure of the energy transformed to radiation during collision is

$$
\Delta E=\frac{E_{i}-E_{f}}{E_{i}},
$$

where $E_{i}$ is the energy of the incoming solitons and $E_{f}$ is the energy of the outgoing ones. The energy $E_{f}$ of the outgoing solitons is inferred by measuring their velocity. As seen in Fig. 11 $\Delta E$ reaches a maximum $5 \%$ for intermediate velocities $v \approx 0.5 v_{s}\left(v_{s}=0.95\right)$.

In Fig. [3 we present five snapshots of the simulation for solitons with initial velocity $v=0.2\left(0.21 v_{s}\right)$. The solitons decelerate as they approach and interact, they reach a minimum separation and finally bounce back. This behaviour is typical for small velocities. One of the initial solitons is denoted by a filled circle in Fig. 2 a , and it appears that it effectively moves along the dispersion curve due to the collision process to the point denoted by an open circle at almost the same energy level. This 
picture gives a precise result for the final outcome of the collision, and it also describes gross features of the collision process. It cannot give the detailed features of the process because the dispersion pertains only to isolated solitary waves. As the velocity of the initial solitons increases, they pass through each other during collision as is seen in Fig. 3 p for $v=0.6\left(0.63 v_{s}\right)$. In the dispersion diagram of Fig. 27 , one of the solitons is represented by a filled square before and by an open square after collision. The energy difference between the two points is small, thus the collision is almost elastic. The overall picture at low $\gamma$ closely resembles the elastic soliton collisions in the integrable NLS [10].

We repeat the simulations for a denser condensate by setting $\gamma=3$ in Eq. (1). Vortex rings, albeit with a very inconspicuous ring structure, form now part of the solitary wave family. The energy radiated during collision reaches now a maximum $23 \%$ at $v=0.4 v_{s}\left(v_{s}=1.29\right)$ as shown in Fig. 1. Nevertheless, collisions for slow and for fast solitary waves are also in this case elastic and their behaviour resembles that for $\gamma=1$ 19]. In Fig. 31 we show five snapshots of the simulation for $v=0.4\left(0.31 v_{s}\right)$. The second snapshot shows the formation of fully fledged vortex rings at the time of collision which do not exist as isolated solitary waves. In fact we have noticed the transient formation of vorticity during solitary-wave collisions in many cases in this work. Similar observations were also made in the simulations of the Ginsberg experiment 9] and for simulated collisions of rarefaction pulses with vortex lines in the homogeneous BEC [15]. The outgoing waves are seen to be of the soliton type in the fourth snapshot. However, these are later spontaneously transformed to depletion droplets, i.e. strongly localised regions of low density, as seen in the last snapshot. At later times not shown in the figure, the droplets transform into soliton-like structures and periodically revive two to three times until we can no longer follow the simulation due to lattice limitations. The speed of the wave is almost constant at $0.48 v_{s}$.

We further study how the picture changes as the coupling increases. The energy-momentum dispersion for axially symmetric solitary waves changes substantially for $\gamma \geq 4$, and it is shown for $\gamma=7$ in Fig. 2b 8]. It has two fundamental branches (solid lines in the figure) which are related by Eq. (3), and they both contain axially symmetric vortex rings with velocities of both signs. For example, for the branch originating on the left we have $1.61=v_{s} \geq v \geq-v_{1}=-0.30 v_{s}$ where $-v_{1}$ is the velocity at the cusp. For velocities close to $v_{s}$, the solitary waves have the structure of gray solitons. For lower velocities they are vortex rings whose radius decreases as $v$ decreases (as $v \rightarrow-v_{1}$ ). In the following we need to distinguish between the vortex rings on the left and those on the right of the dispersion maximum. We call the vortex rings with $v>0$ type I, and those with $-v_{1} \leq v<0$ type II. Finally, the symmetry relation (3) gives a second

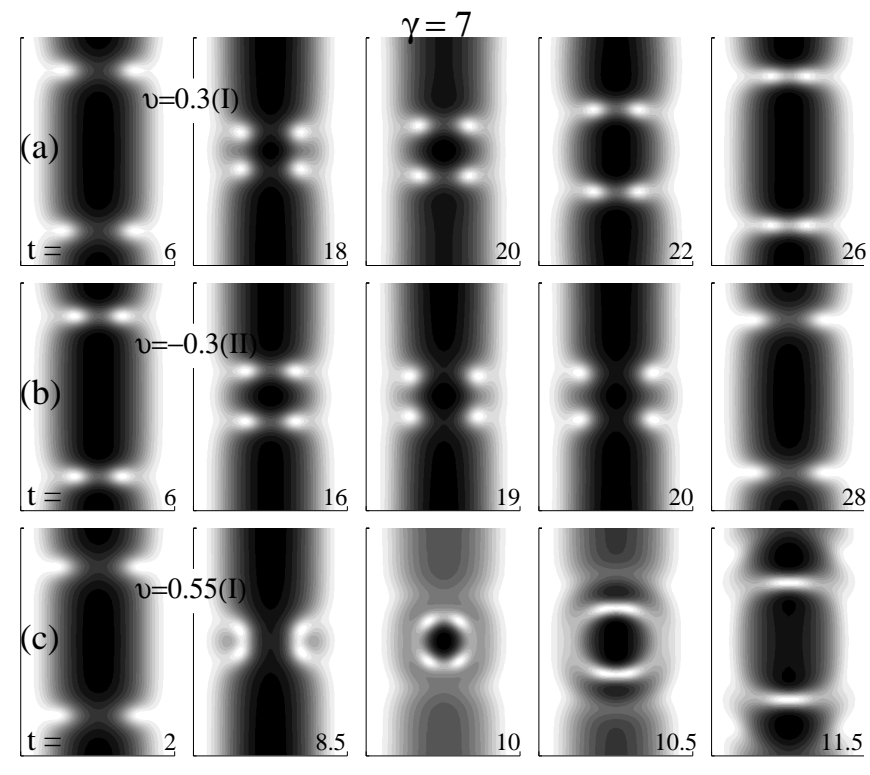

FIG. 4: Particle density $|\Psi|^{2}$ for solitary wave collisions for $\gamma=7$. The frames are $8 \times 12$.

branch in Fig. 2b with mirror imaged type I and II rings. The branch denoted by a dotted line in Fig. 20 contains unstable solitary waves of higher energy and will not be discussed further here.

We simulate the collision of two counter-propagating vortex rings of opposite circulation related by Eq. (3). We first discretized the wave function in a $3 \mathrm{D}$ lattice in Cartesian coordinates. After checking in test runs that the axial symmetry was not broken during collision we proceeded to extensive simulations assuming axial symmetry throughout the process. The energy radiated due to collision for a coupling constant $\gamma=7$ is shown in Fig. 1] The maximum is $60 \%$ and occurs for initial velocity $v=0.37 v_{s},\left(v_{s}=1.61\right)$. On the other hand, collisions at low and high velocities appear to be elastic, i.e., the outgoing solitary waves have almost the same energy as the incoming ones. The three curves in Fig. 11 for $\gamma=1,3$, and 7 show that collisions are elastic for all couplings when the colliding solitary waves are slow or when their velocity is close to the speed of sound [19]. For intermediate velocities energy is radiated during collision and this is higher as the coupling increases.

Fig. 4. shows the collision of vortex rings with an initial velocity $v=0.3\left(0.19 v_{s}\right)$. The initial rings are denoted by a filled circle in the dispersion (Fig. 2b) and they are type I. They reach a minimum distance, their radius decreases, and subsequently they bounce back. The outgoing rings of type II still have the original circulation and are denoted by an open circle on the dispersion curve. The change in nature of the rings is apparent in the change of the ring radius between the first and last snapshots in Fig. 4a. The energy radiated during this process is $3 \%$. It appears that each ring has effectively moved along one of 
the branches of the dispersion curve. This picture gives a precise result for the final outcome of the collision, but it also gives a faithful representation of the gross features throughout the collision process. The almost elastic collisions of slow vortex rings seen here are in stark contrast to collisions in the $3 \mathrm{D}$ bulk where vortex rings increase their radius and eventually annihilate 12 . They are inhibited to do this in our model due to the transverse confinement.

We may choose the initial vortex rings to be type II but still have initial velocity $|v|=0.3\left(0.19 v_{s}\right)$ (Fig. 4b). In this case the collision causes them to increase their radius, and they are eventually transformed to type I rings moving in opposite directions. The process is essentially the reverse of the one in Fig. 4 a.

The arguments pertaining to the dispersion curve can be employed to rationalise the observed behaviour for higher velocities (lower energies). One can thus imagine a collision where the initial type I vortex rings have energy slightly lower than the energy at the cusp, as for example at the point denoted by a filled square in Fig. 20 (corresponding to $\left.v=0.5\left(0.31 v_{s}\right)\right)$. The bounce-back process of vortex rings cannot occur in this case. Instead, intermediate structures are formed, as discussed below, and the collisions become highly inelastic. In the example of Fig. 20 the final waves, denoted by an open square, have substantially lower energy than the initial rings. This mechanism of inelastic collision suggests that the maximum energy loss occurs at intermediate velocities.

A further example of a collision for an initial velocity $0.55\left(0.34 v_{s}\right)$ for type I rings is shown in Fig. 4t. The vortex rings form a shell-like object of low density as they collide, as seen in the third entry of the figure. Since we have assumed axial symmetry, the shell is actually almost spherical, and strongly reminiscent of those observed in Ref. 9]. However, the shells reported here are produced by an elementary head-on collision process which is significantly simpler than the process reported in the experiment where many nonlinear wave structures interact simultaneously while the condensate is expanding. The resemblance between the observed structures suggests that inelastic collisions of axisymmetric nonlinear waves is the fundamental process underlying the generation of spherical waves observed experimentally [9]

As the rings move away to opposite directions they form depletion droplets (similar to those seen in Fig. 3.), which are shown in the last entry of the figure. These travel coherently for a distance of approximately 10 units and they eventually seem to decay into solitons. The depletion droplets are distinctly different than solitary waves theoretically studied in a confined BEC but they are reminiscent of the rarefaction pulses in the homogeneous Bose gas [11, 18].

The collision in Fig. 4p is highly inelastic in the sense that the main outgoing waves carry only part of the total energy while the rest of the energy is radiated away.
However, it is possible that some of the remaining energy is carried by shallow gray solitons.

At small energies ( $v$ close to $v_{s}$ ) the solitary waves have a gray-soliton character. They pass through each other during collision much like the situation for small couplings. In Fig. 20 the solitary wave with $v=1.0\left(0.62 v_{s}\right)$ is denoted by a filled triangle. After the collision it has only slightly lower energy (open triangle). Thus, collisions for large velocities are elastic as is also shown in Fig. 1 [1]].

Concluding, we have shown that elastic collisions of solitary waves can occur in cylindrical BECs. Shell structures reminiscent of recent experimental observations were shown to arise already in elementary inelastic headon collisions. Possible extensions of the present work to larger densities, non-axisymmetric solitary waves, and beyond the head-on case will provide further valuable insights into the dynamics of nonlinear waves in confined BECs.

We thank L. Hau, N. Ginsberg, N. Berloff and N. Papanicolaou for inspiring discussions. This work was supported by EPSRC Grant No GR/R96026/01, and has benefited from a visit to KITP, Santa Barbara (SK).

[1] S. Burger, et al, Phys. Rev. Lett. 83, 5198 (1999).

[2] J. Denschlag, et al, Science 287, 97 (2000).

[3] Z. Dutton, et al, Science, 293, 663 (2001).

[4] B.P. Anderson, et al, Phys. Rev. Lett. 86, 2926 (2001).

[5] D.L. Feder, et al, Phys. Rev. A 62, 053606 (2000).

[6] J. Brand and W. P. Reinhardt, J. Phys. B 34, L113 (2001); Phys. Rev. A 65, 043612 (2002).

[7] S. Komineas and N. Papanicolaou, Phys. Rev. Lett. 89, 070402 (2002); Phys. Rev. A 67, 023615 (2003).

[8] S. Komineas and N. Papanicolaou, Phys. Rev. A 68, 043617 (2003).

[9] N. S. Ginsberg, J. Brand, and L. V. Hau, Phys. Rev. Lett. 94, 040403 (2005).

[10] M.J. Ablowitz, H. Segur, Solitons and the inverse scattering transform (SIAM, 1981).

[11] C.A. Jones and P.H. Roberts, J. Phys. A: Math. Gen. 15, 2599 (1982).

[12] J. Koplik and H. Levine, Phys. Rev. Lett. 76, 4745 (1996).

[13] N.G. Berloff, J. Phys. A 37, 1617 (2004).

[14] M. Leadbeater et al., Phys. Rev. Lett. 86, 1410 (2001); Phys. Rev. A 67, 015601 (2003).

[15] N.G. Berloff, Phys. Rev. A 69, 053601 (2004).

[16] L.D. Carr, M.A. Leung, and W.P. Reinhardt, J. Phys. B 33, 3983 (2000).

[17] T. Schulte, et al., Phys. Rev. A 66, 033602 (2002).

[18] Inelastic collisions of small vortex-rings on a homogeneous background were found to result in the formation of rarefaction pulses, N. Berloff, private communication.

[19] The numerically determined value of $\Delta E$ is very small for $v \approx v_{s}$, but it cannot be calculated very accurately because $E_{i}$ and $E_{f}$ are small. 